PROCEEDINGS OF THE

AMERICAN MATHEMATICAL SOCIETY

Volume 133, Number 11, Pages 3191-3197

S 0002-9939(05)07887-1

Article electronically published on May 9, 2005

\title{
THE ESSENTIAL IDEAL IS A COHEN-MACAULAY MODULE
}

\author{
DAVID J. GREEN
}

(Communicated by Paul Goerss)

\begin{abstract}
Let $G$ be a finite $p$-group which does not contain a rank two elementary abelian $p$-group as a direct factor. Then the ideal of essential classes in the mod- $p$ cohomology ring of $G$ is a Cohen-Macaulay module whose Krull dimension is the $p$-rank of the centre of $G$. This basically answers in the affirmative a question posed by J. F. Carlson (Question 5.4 in Problems in the calculation of group cohomology, 1999).
\end{abstract}

\section{INTRODUCTION}

Let $G$ be a finite $p$-group and let $k$ be a field of characteristic $p$. By definition, the essential ideal $\operatorname{Ess}(G)$ in the cohomology ring $H^{*}(G, k)$ consists of those cohomology classes whose restriction to every proper subgroup is zero. The essential ideal is involved in the cohomological characterization of $p$-groups whose exponent $p$ elements are all central [1, and it has featured prominently in investigations concerning the depth of the graded commutative ring $H^{*}(G, k)$ (see [7).

The essential ideal also plays a key role in Carlson's method 9] for computing $H^{*}(G, k)$. In this paper we shall show that the most important case of a question posed by Carlson (Question 5.4 in [8]) can be answered using existing techniques. That is, we shall prove the following theorem:

Theorem A. Let $k$ be a field of characteristic $p$, and let $G$ be a finite p-group which does not have the elementary abelian p-group of order $p^{2}$ as a direct factor. If the essential ideal $\operatorname{Ess}(G)$ in $H^{*}(G, k)$ is nonzero, then it is a Cohen-Macaulay module with Krull dimension equal to the p-rank of the centre of $G$.

Another formulation of the result is as follows:

Theorem B. With the assumptions of Theorem $\mathrm{A}$, denote by $C$ the largest central elementary abelian subgroup of $G$. Suppose that $\zeta_{1}, \ldots, \zeta_{d}$ are homogeneous elements of $H^{*}(G, k)$ such that the restrictions $\operatorname{Res}_{C}\left(\zeta_{1}\right), \ldots, \operatorname{Res}_{C}\left(\zeta_{d}\right)$ form a homogeneous system of parameters for $H^{*}(C, k)$. Then the essential ideal $\operatorname{Ess}(G)$ in $H^{*}(G, k)$ is free and finitely generated as a module over the polynomial algebra $k\left[\zeta_{1}, \ldots, \zeta_{d}\right]$.

Carlson's method for computing $H^{*}(G, k)$ depends upon the essential ideal being free and finitely generated. Up until now the computer program had to check this property for each group it was presented with. Now that the question is a theorem,

Received by the editors February 27, 2004 and, in revised form, June 24, 2004.

2000 Mathematics Subject Classification. Primary 20J06; Secondary 13C14.

(C)2005 American Mathematical Society

Reverts to public domain 28 years from publication 
programs based on Carlson's method should run faster. Note that the same question was also mentioned by H. Mui several years earlier, but in a much weaker form [12].

The equivalence of Theorems $\mathrm{A}$ and $\mathrm{B}$ is demonstrated in Lemma 1.3. which also shows that it suffices to find one sequence $\zeta_{1}, \ldots, \zeta_{d}$ satisfying the assumptions and the conclusion of Theorem B Finite generation of $\operatorname{Ess}(G)$ is proved in Lemma 1.2 for all finite $p$-groups $G$; and freeness is proved for the groups under consideration in $\$ 2$, using the fact that the cohomology ring of $G$ is a comodule over the cohomology ring of any central subgroup. From now on we shall write $H^{*}(G)$ for $H^{*}(G, k)$.

\section{Group Cohomology And Commutative Algebra}

We shall demonstrate that Theorems $\mathrm{A}$ and $\mathrm{B}$ are equivalent by recalling several well-documented facts about group cohomology and commutative algebra. The following lemma is certainly not new.

Lemma 1.1. Let $k$ be a field of characteristic $p$ and let $G$ be a finite p-group whose essential ideal $\operatorname{Ess}(G)$ is nonzero. Denote by $C$ the largest central elementary abelian subgroup of $G$. Let $A, I$ and $J$ be the following ideals in $H^{*}(G)$ :

- $A$ is the annihilator ideal $\operatorname{Ann}_{H^{*}(G)}(\operatorname{Ess}(G))$;

- $I$ is the kernel of restriction $H^{*}(G) \rightarrow H^{*}(C)$; and

- $J$ is generated by all transfer classes from all proper subgroups.

Then $\sqrt{A}=\sqrt{I}=\sqrt{J}$, and this radical ideal is a prime ideal.

Proof. The cohomology ring of an elementary abelian $p$-group is a polynomial algebra $(p=2)$ or the tensor product over $k$ of a polynomial algebra with an exterior algebra $\left(p\right.$ odd). So $H^{*}(C) / \sqrt{0}$ is a polynomial algebra, and $\sqrt{I}$ a prime ideal. Quillen proved that $x \in H^{*}(G)$ is nilpotent if its restriction to every elementary abelian subgroup is nilpotent (Coroll. 8.3.4 in [10]). So $\sqrt{I}$ consists of those classes with nilpotent restriction to the centre $Z(G)$. It follows from Carlson's theorem on varieties and transfer $(\S 10.2$ in [10]) that $\sqrt{I}=\sqrt{J}$.

Let $H \leq G$ be a subgroup. Then restriction makes $H^{*}(H)$ a module over $H^{*}(G)$, and transfer from $H^{*}(H)$ to $H^{*}(G)$ is a homomorphism of $H^{*}(G)$-modules. Hence transfer classes and essential classes annihilate each other, which means that $J \subseteq A$.

To finish the proof we will use an argument of Broto and Henn [6] to show that every element of $H^{*}(G) \backslash \sqrt{I}$ is regular, from which it follows that $A \subseteq \sqrt{I}$. Group multiplication $\mu: G \times C \rightarrow G$ is a group homomorphism, and so induces a map of $k$-algebras $\mu^{*}: H^{*}(G) \rightarrow H^{*}(G) \otimes_{k} H^{*}(C)$. Moreover, $\left(\operatorname{Id}_{G} \times \varepsilon_{C}\right) \circ \mu^{*}=\operatorname{Id}_{G}$ and $\left(\varepsilon_{G} \otimes \operatorname{Id}_{C}\right) \circ \mu^{*}=\operatorname{Res}_{C}^{G}$, where $\operatorname{Id}_{G}: H^{*}(G) \rightarrow H^{*}(G)$ is the identity map and $\varepsilon_{G}: H^{*}(G) \rightarrow k$ the augmentation. So $\mu^{*}$ is a split monomorphism, and $\mu^{*}(x) \in 1 \otimes \operatorname{Res}_{C}(x)+H^{>0}(G) \otimes H^{*}(C)$ for all $x \in H^{*}(G)$. So if $\operatorname{Res}_{C}(x)$ is nonnilpotent, then it is a regular element of $H^{*}(C)$, and it follows that $\mu^{*}(x)$ is a regular element of $H^{*}(G) \otimes_{k} H^{*}(C)$. As $\mu^{*}$ is injective, it follows that $x \in H^{*}(G)$ is regular.

Lemma 1.2. Let $G$ be a finite $p$-group, and let $k$ be a field of characteristic $p$. Denote by $C$ the largest central elementary abelian subgroup of $G$. Suppose that $\zeta_{1}, \ldots, \zeta_{d}$ are homogeneous elements of $H^{*}(G)$ whose restrictions to $C$ form a homogeneous system of parameters for $H^{*}(C)$. Then $\operatorname{Ess}(G)$ is finitely generated as a module over the polynomial algebra $R=k\left[\zeta_{1}, \ldots, \zeta_{d}\right]$. 
Proof. The Evens-Venkov Theorem (Coroll. 7.4.6 in [10]) states that $H^{*}(G)$ is a finitely generated $k$-algebra. Let $A, I, J$ be as in Lemma 1.1] and recall from the proof of that lemma that $J \subseteq A$. So $\operatorname{Ess}(G)$ is a module over $H^{*}(G) / J$, and it is finitely generated since $H^{*}(G)$ is noetherian. So it would suffice to show that $H^{*}(G) / J$ is a finitely generated $R$-module.

If $S \subseteq T$ are finitely generated (graded-)commutative $k$-algebras and $L \subseteq T$ is an ideal such that $T / \sqrt{L}$ is a finitely generated $S$-module, then $T / L$ is a finitely generated $S$-module, too. So it suffices to show that $H^{*}(G) / \sqrt{J}$ is a finitely generated $R$-module. But we have seen that $\sqrt{J}=\sqrt{I}$, and by assumption $H^{*}(G) / I$ is a submodule of the noetherian $R$-module $H^{*}(C)$.

Lemma 1.3. Let $G$ be a finite p-group and let $k$ be a field of characteristic $p$. Denote by $C$ the largest central elementary abelian subgroup of $G$. Assuming that $\operatorname{Ess}(G)$ is nonzero, the following three statements are equivalent:

(1) $\operatorname{Ess}(G)$ is a Cohen-Macaulay $H^{*}(G)$-module.

(2) There is a sequence $\zeta_{1}, \ldots, \zeta_{d}$ of homogeneous elements of $H^{*}(G)$ such that:

(a) the restrictions $\operatorname{Res}_{C}\left(\zeta_{1}\right), \ldots, \operatorname{Res}_{C}\left(\zeta_{d}\right)$ form a homogeneous system of parameters for $H^{*}(C)$; and

(b) the essential ideal $\operatorname{Ess}(G)$ is free and finitely generated as a module over the polynomial algebra $k\left[\zeta_{1}, \ldots, \zeta_{d}\right]$.

(3) Every sequence $\zeta_{1}, \ldots, \zeta_{d}$ which satisfies condition (2a) also satisfies condition (2b).

If these equivalent conditions hold, then $d$ and the Krull dimension of the module $\operatorname{Ess}(G)$ both coincide with the p-rank of the centre of $G$.

Proof. Let $A, I, J$ be as in Lemma 1.1. First we shall see that condition (2a) is equivalent to the following condition:

(2a) The images of $\zeta_{1}, \ldots, \zeta_{d}$ in $H^{*}(G) / A$ are algebraically independent over $k$, and $\operatorname{Ess}(G)$ is a finitely generated module over $R=k\left[\zeta_{1}, \ldots, \zeta_{d}\right]$.

Since $\sqrt{I}=\sqrt{A}$, the images of $\zeta_{1}, \ldots, \zeta_{d}$ are algebraically independent in $H^{*}(G) / A$ if and only if they are algebraically independent in $H^{*}(G) / I \subseteq H^{*}(C)$. Lemma 1.2 shows that (2a) implies that $\operatorname{Ess}(G)$ is a finitely generated $R$-module. Conversely, (2a) implies that $\operatorname{Ess}(G)$ and therefore $H^{*}(G) / A$ are finitely generated $R$-modules. Since $\sqrt{I}=\sqrt{A}$, it follows that $H^{*}(G) / \sqrt{I}$ is a finitely generated $R$-module. So just as in the proof of Lemma 1.2, $H^{*}(G) / I$ is a finitely generated $R$-module. But recall from Corollary 7.4.7 of [10] that $H^{*}(C)$ is finite over the image $H^{*}(G) / I$ of restriction from $H^{*}(G)$. So $H^{*}(C)$ is a finitely generated $R$-module. Hence (2a) and (2a) are indeed equivalent.

But the characterisation of Cohen-Macaulay modules in Theorem 4.3.5 of [3] states precisely that (11), (22) and (3i) are equivalent if one replaces (2a) by (2a) in (2) and (3). Moreover, $d=\operatorname{dim}(\operatorname{Ess}(G))$ would follow, too. As $H^{*}(C)$ is polynomial for $p=2$ and polynomial tensor exterior for $p$ odd, and the dimension is equal to the rank of $C$ in both cases, $d$ is the $p$-rank of the centre of $G$.

Remark. Assuming that the equivalent conditions (2a), (2a) hold, apply the same characterisation of Cohen-Macaulay modules to $H^{*}(C)$. Recalling that $H^{*}(C)$ is a polynomial algebra for $p=2$, and polynomial tensor exterior for $p$ odd, one deduces that $H^{*}(C)$ is a free and finitely generated $R$-module. 


\section{Freeness}

As in [6], observe that group multiplication $\mu: G \times C \rightarrow G$ turns $H^{*}(G)$ into a comodule over the coalgebra $H^{*}(C)$. As was noted above, the comodule structure map $\mu^{*}: H^{*}(G) \rightarrow H^{*}(G) \otimes_{k} H^{*}(C)$ is simultaneously a map of $k$-algebras.

The following lemma is the key to this paper. It is perhaps the natural level of generality for a result that has been known in various special cases for some time (Lemma 3.1 in [2, Proposition 5.2 in 8 and Lemma 1.2 in [1]). The lemma bears a striking resemblance to a classical fact about Hopf algebras (Theorem 4.1.1 in 13 ), but I cannot see a way to derive the former as a corollary of the latter.

Lemma 2.1. Let $G, C, R$ be as in Lemma[1.2, and let $F=\bigoplus_{i \geq i_{0}} F_{i}$ be the free graded $H^{*}(G)$-module on generators $e_{1}, \ldots, e_{s}$ (not necessarily in degree zero). Hence $F$ is a comodule over $H^{*}(C)$, with structure map $\psi$ given by $\psi\left(a e_{j}\right)=$ $\mu^{*}(a) \cdot\left(e_{j} \otimes 1\right) \in F \otimes_{k} H^{*}(C)$. Let $N \subseteq M$ be graded submodules of $F$ which are simultaneously subcomodules. Then $M / N$ is a free $R$-module.

Proof. Define $\bar{\psi}: M / N \rightarrow M / N \otimes_{k} H^{*}(C)$ by $\bar{\psi}(a+N)=\psi(a)+N \otimes_{k} H^{*}(C)$. By the assumptions, $(M / N, \bar{\psi})$ is an $H^{*}(C)$-comodule. Moreover $\bar{\psi}$ is a map of $H^{*}(G)$ modules if one gives $M / N \otimes_{k} H^{*}(C)$ the $H^{*}(G)$-module structure induced from the obvious $H^{*}(G) \otimes_{k} H^{*}(C)$-module structure by $\mu^{*}$. In fact, $\bar{\psi}$ is a split monomorphism of $H^{*}(G)$-modules, the splitting map being $\operatorname{Id}_{M / N} \otimes \varepsilon$, where $\varepsilon: H^{*}(C) \rightarrow k$ is the augmentation map.

Inclusion of $R$ in $H^{*}(G)$ induces an $R$-module structure on $M / N \otimes_{k} H^{*}(C)$. We shall show that this $R$-module is free. It then follows that $\zeta_{1}, \ldots, \zeta_{d}$ is a regular sequence for this $R$-module, and therefore for the $R$-module $M / N$, too. Hence $M / N$ is a free $R$-module.

For $i \geq i_{0}$ set $S_{i}:=\bigoplus_{j \geq i}(M / N)_{i}$, and let $T_{i}$ be the $R$-submodule $S_{i} \otimes_{k} H^{*}(C)$ of $M / N \otimes H^{*}(C)$. Then $M / N \otimes_{k} H^{*}(C)=T_{i_{0}} \supseteq T_{i_{0}+1} \supseteq T_{i_{0}+2} \supseteq \cdots$ and $\bigcap_{i \geq i_{0}} T_{i}=\{0\}$. Now, $T_{i} / T_{i+1}$ is $H^{*}(C)$-module, and as such it is free of rank $\operatorname{dim}_{k}(M / N)_{i}$. Moreover the $R$-module structure is induced from this $H^{*}(C)$-module structure by inclusion $R \hookrightarrow H^{*}(G)$ followed by restriction to $H^{*}(C)$, since for all $\rho \in R, x \in S_{i}$ and $y \in H^{*}(C)$ one has $\rho \cdot\left(x \otimes y+T_{i+1}\right)=x \otimes \operatorname{Res}_{C}(\rho) \cdot y+T_{i+1}$. We observed above that $H^{*}(C)$ is a free $R$-module, and so $T_{i} / T_{i+1}$ is a free $R$-module. By induction, $T_{i_{0}} / T_{i}$ is a free $R$-module for all $i$. Since $T_{i}$ is confined to degree $\geq i$, this means that $T_{i_{0}}=M / N \otimes_{k} H^{*}(C)$ is itself a free $R$-module.

Lemma 2.2. In Lemma 1.3 there is always a sequence $\zeta_{1}, \ldots, \zeta_{d}$ satisfying condition (2a).

Proof. Restriction turns $H^{*}(C)$ into an $H^{*}(G)$-module; recall from Corollary 7.4.7 of [10] that this module is finitely generated. The result follows by the graded case of Noether Normalization (Theorem 2.2.7 in [3]) applied to the $H^{*}(G)$-module $H^{*}(C)$ : recall that the annihilator ideal of this $H^{*}(G)$-module is the kernel of the restriction map.

One explicit sequence is $\zeta_{i}=m$ th Chern class of the regular representation of $G$, for $m=2\left(p^{n}-p^{n-i}\right)$, where $|G|=p^{n}$. This construction features in Venkov's topological proof of the Evens-Venkov theorem (§3.10 in [4]).

Proof of Theorems $\mathrm{A}$ and $\mathrm{B}$. Lemma 1.3 shows that the two theorems are equivalent, and that to prove them it suffices to prove the existence of a sequence $\zeta_{1}, \ldots, \zeta_{d}$ 
satisfying conditions (2a) and (2b). Lemma 2.2 demonstrates that there is always a sequence satisfying (2a), and by Lemma 1.2 the finite generation part of (2b) is an automatic consequence of (2a). We shall adopt the notation of Lemma 1.2.

Assume first that the cyclic group of order $p$ is not a direct factor of $G$. Then every maximal subgroup of $G$ contains $C$, and so $\operatorname{Ess}(G)$ is a subcomodule of the $H^{*}(C)$-comodule $H^{*}(G)$. Hence $\operatorname{Ess}(G)$ is a free $R$-module by Lemma 2.1 so the theorems are proved for this $G$.

Now suppose that $G=H \times C_{p}$, and that $H$ has no direct factor which is cyclic of order $p$. Denote by $C^{\prime}$ the largest central elementary abelian subgroup of $H$. Then $C=C^{\prime} \times C_{p}$, and $\operatorname{Ess}(H)$ is a $H^{*}\left(C^{\prime}\right)$-comodule by the above argument. Applying Lemma 2.2 to $H, C^{\prime}$, one obtains a sequence $\zeta_{1}, \ldots, \zeta_{d-1} \in H^{*}(H)$ which satisfies condition (2a) of Lemma 1.3 for $H, C^{\prime}$. Let $t$ be a homogeneous system of parameters for the one-dimensional $k$-algebra $H^{*}\left(C_{p}\right)$. Then $\zeta_{1}, \ldots, \zeta_{d-1}, t$ satisfies condition (2a) for $G, C$. Set $S=k\left[\zeta_{1}, \ldots, \zeta_{d-1}\right] \subseteq H^{*}(H)$ and $R=S[t] \subseteq H^{*}(G)$. Observe that $\operatorname{Ess}(H)$ is a free $S$-module by the case we have already proved. Let $b_{1}, \ldots, b_{n}$ be a finite free generating set of the $k[t]$-module $H^{*}\left(C_{p}\right)$. We take each $b_{\ell}$ to be homogeneous. Let $B \subseteq H^{*}\left(C_{p}\right)$ be the $k$-vector space with basis $b_{1}, \ldots, b_{n}$. Then $H^{*}\left(C_{p}\right)=\bigoplus_{j \geq 0} t^{j} B$.

Set $F_{i}:=H^{*}(H) \otimes_{k} \bigoplus_{j=0}^{i} t^{j} B$. Define $M_{i}$ and $N_{i}$ by $M_{i}:=\operatorname{Ess}(G) \cap F_{i}$ and $N_{i}:=M_{i-1}+M_{i-1} t$. Both $M_{i}$ and $N_{i}$ are submodules of the free and finitely generated $H^{*}(H)$-module $F_{i}$. Moreover they are subcomodules of the $H^{*}\left(C^{\prime}\right)$ comodule $F_{i}$, as $C^{\prime}$ lies in every maximal subgroup of $G$. So $M_{i} / N_{i}$ is a free $S$ module by Lemma 2.1. Observe that the $R$-modules $k[t] N_{i}$ and $k[t] M_{i-1}$ coincide. Moreover, the noetherian $R$-module $\operatorname{Ess}(G)$ is the union of the $R$-modules $k[t] M_{i}$, and so $k[t] M_{i}=\operatorname{Ess}(G)$ for large enough $i$.

It would suffice to show that each $k[t] M_{i}$ is a free $R$-module, and this follows by induction if we can show that each $\left(k[t] M_{i}\right) /\left(k[t] N_{i}\right)$ is a free $R$-module. (Note that $N_{0}=\{0\}$.) We shall show this by proving that $\left(k[t] M_{i}\right) /\left(k[t] N_{i}\right)$ is the free $S[t]$-module $k[t] \otimes_{k}\left(M_{i} / N_{i}\right)$.

Note that $\operatorname{Ess}(G) \subseteq \operatorname{Ess}(H) \otimes_{k} H^{*}\left(C_{p}\right)$, since $K \times C_{p}$ is a maximal subgroup of $G$ for each maximal subgroup $K$ of $H$. (For $H=1$ one has $\operatorname{Ess}(1)=k=H^{*}(1)$.)

Let $y$ be an element of $M_{i}$ in degree $m$. Then there are homogeneous classes $a_{j \ell} \in \operatorname{Ess}(H)$ for $0 \leq j \leq i$ and $1 \leq \ell \leq n$ with $y=\sum_{j=0}^{i} \sum_{\ell=1}^{n} a_{j \ell} \otimes b_{\ell} t^{j}$. Call $\sum_{\ell} a_{i \ell} \otimes b_{\ell}$ the leading coefficient of $y$, and let $I_{i} \subseteq \operatorname{Ess}(H) \otimes_{k} B$ denote the set of all such leading coefficients. Then $I_{i}$ is an $H^{*}(H)$-module, and $y$ lies in $N_{i}$ if and only if its leading coefficient lies in $I_{i-1}$. So we may pick a basis for a complement of the subspace $N_{i}$ of $M_{i}$ consisting of classes whose leading terms constitute a basis for a complement of the subspace $I_{i-1}$ of $I_{i}$. So the natural surjective map of $S[t]$-modules $k[t] \otimes_{k}\left(M_{i} / N_{i}\right) \rightarrow\left(k[t] M_{i}\right) /\left(k[t] N_{i}\right)$ is injective, too. But the domain is a free $S[t]$-module.

\section{Concluding Remarks}

Application to computer calculations. In [9], Carlson describes a series of tests on a partial presentation for $H^{*}(G)$ and proves that the presentation is complete if it passes the tests. This is of crucial importance for the computer calculation of group cohomology via minimal resolutions, for otherwise one would never know when to stop. 
However Carlson's tests depend on two conjectures about the structure of the cohomology ring, which have to be checked for the group in question as part of the calculation. This means that there could conceivably be some groups where there is no complete presentation that the tests can detect as being complete. One of these conjectures concerns the Koszul complex associated to a homogeneous system of parameters. The other is now proven, as our Theorem B, note that Carlson's tests assume that the cohomology rings of all subgroups are known, and so the cohomology ring of a product group is known by the Künneth theorem.

Benson's test for completion. Benson recently obtained a new test for completion (Theorem 10.1 of [5]). Unlike Carlson's test, Benson's test does not depend on any conjectures. What is more, it offers the prospect of calculating the cohomology ring from a smaller initial segment of the minimal resolution than Carlson's test. Hence implementing Benson's test should speed up calculations appreciably.

Note that the result of this paper also has the potential to speed up calculations based on Benson's test. For example, the Sylow 2-subgroup of the symmetric group $S_{8}$ has order 128 and 2-rank 4; and the 2-rank of its centre is one. All relations and generators in the cohomology ring occur by degree six. For Carlson's test one has to calculate out to degree 12, whereas for Benson's test one has to calculate out to degree 9. As the essential ideal is zero for this group, one knows at the end of degree 6 that there can be no new relations among the existing generators. So after degree 6 it is enough to calculate the dimensions of the cohomology groups: one does not have to perform the time-consuming cochain lifts needed to evaluate products. The result of this paper means that one can use the same shortcut in the presence of essential classes.

\section{REFERENCES}

[1] Alejandro Adem and Dikran Karagueuzian, Essential cohomology of finite groups, Comment. Math. Helv. 72 (1997), no. 1, 101-109. MR1456319|(98f:20038)

[2] Alejandro Adem and R. James Milgram, The mod 2 cohomology rings of rank 3 simple groups are Cohen-Macaulay, Prospects in topology (Princeton, NJ, 1994) (Frank Quinn, ed.), Ann. of Math. Stud., vol. 138, Princeton Univ. Press, Princeton, NJ, 1995, pp. 3-12. MR1368650 (96m:20084)

[3] D. J. Benson, Polynomial invariants of finite groups, London Math. Soc. Lecture Note Series, vol. 190, Cambridge University Press, Cambridge, 1993. MR1249931 (94j:13003)

[4] _ Representations and cohomology. II, second ed., Cambridge Studies in Advanced Math., vol. 31, Cambridge University Press, Cambridge, 1998. MR.1634407 (99f:20001b)

[5] _ Dickson invariants, regularity and computation in group cohomology, Illinois J. Math. 48 (2004), no. 1, 171-197. MR2048221

[6] Carlos Broto and Hans-Werner Henn, Some remarks on central elementary abelian psubgroups and cohomology of classifying spaces, Quart. J. Math. Oxford Ser. (2) 44 (1993), no. 174, 155-163. MR1222371 (94c:57060)

[7] Jon F. Carlson, Depth and transfer maps in the cohomology of groups, Math. Z. 218 (1995), no. 3, 461-468. MR 1324540 (95m:20058)

[8] _ Problems in the calculation of group cohomology, Computational methods for representations of groups and algebras (Essen, 1997) (P. Dräxler, G. O. Michler, and C. M. Ringel, eds.), Birkhäuser, Basel, 1999, pp. 107-120. MR1714605 (2001i:20111)

[9] Calculating group cohomology: Tests for completion, J. Symbolic Comput. 31 (2001), no. 1-2, 229-242. MR.1806218 (2002c:20083)

[10] Leonard Evens, The cohomology of groups, Oxford Univ. Press, Oxford, 1991. MR 1144017 (93i:20059)

[11] David J. Green, On Carlson's depth conjecture in group cohomology, Math. Z. 244 (2003), no. 4, 711-723. Mr2000456(2004h:20072) 
[12] Huỳnh Mui, The mod $p$ cohomology algebra of the extra-special group E( $\left.p^{3}\right)$, Unpublished essay, 1982.

[13] Moss E. Sweedler, Hopf algebras, Mathematics Lecture Note Series, W. A. Benjamin, Inc., New York, 1969. MR0252485|(40:5705)

Department of Mathematics, University of Wuppertal, D-42097 Wuppertal, Germany

E-mail address: green@math.uni-wuppertal.de 